\title{
Leopoldo Zea en Puerto Rico
}

\section{Leopoldo Zea in Puerto Rico}

DOI: $10.46814 / 1 a j d v 3 n 4-077$

Recebimento dos originais: 01/05/2021

Aceitação para publicação: 31/06/2021

Vivian Auffant-Vázquez

Profesora, investigadora, ensayista y escritora puertorriqueña.

Doctora en Filosofía y Letras de Temple University, Filadelfia, Pensilvania especializada en Literatura Colonial Latinoamericana. Tiene Certificado Profesional de Bioética del Recinto de Ciencias Médicas, Universidad de Puerto Rico. Miembro de la Cátedra Internacional de la UNESCO José Martí. Actualmente es Catedrática en la Facultad de Estudios Generales de la Universidad de Puerto Rico en Río Piedras.

\section{RESUMEN}

Esta investigación presenta el contenido temático respecto al Primer Seminario de Historia de las Ideas en Puerto Rico del 3 al 8 de diciembre de 1956 convocado por el Instituto Panamericano de Geografía e Historia, institución que patrocina a la Colección Historia de las Ideas de América la cual dirige el Dr. Leopoldo Zea en 1956. Esta participación intelectual es la primera en Puerto Rico del filósofo mexicano. En ella se enlaza la Isla a la corriente filosófica latinoamericana. Este aspecto lo destacará Zea posteriormente en un artículo del tomo publicado sobre el Sesquicentenario de Eugenio María de Hostos celebrado en 1989.

Palabras claves: filosofía latinoamericana, libertad, derecho, justicia

\section{ABSTRACT}

This research presents the thematic content of the First Seminar on History of Ideas in Puerto Rico from December 3 to 8, 1956, convened by the Pan American Institute of Geography and History, institution that sponsors the Collection History of Ideas of America, directed by Dr. Leopoldo Zea in 1956. This intellectual participation is the first in Puerto Rico of the Mexican philosopher. It links the island to the Latin American philosophical current. Zea will emphasize this aspect later in an article of the volume published on the Sesquicentennial of Eugenio Maria de Hostos celebrated in 1989.

Key words: Latin American philosophy, freedom, law, justice.

\section{INTRODUÇÃO}

La semblanza que José Luis Gómez Martínez hace sobre Leopoldo Zea en el volumen Pensamiento filosófico latinoamericano del Caribe y "latino ${ }^{\prime}(1300-2000)^{l}$ es una de sumo interés para nuestro tema. Destaca que Zea sucede a Antonio Caso en la cátedra de historia de la filosofía en

\footnotetext{
${ }^{1}$ Pensamiento filosófico latinoamericano del Caribe y “latino“(1300-2000), Editores Enrique Dussel, Eduardo Mendieta, Carmen Bohórquez, México, Siglo XXI,2009:839-841.
} 
la UNAM en 1944, y funda en la Facultad de Filosofía de la UNAM el Seminario sobre Historia de las Ideas de América en 1947; a partir de 1952 dirige la Colección México y lo mexicano editado por Porrúa. El esfuerzo de Zea en el desarrollo y difusión de las ideas latinoamericanas, lleva a destacarlo en 1956 y lo nombran director de la Colección Historia de las Ideas de América bajo el patrocinio del Instituto Panamericano de Geografía e Historia. El propósito del Instituto es trabajar sobre el desarrollo de las ideas en Latinoamérica.

Leopoldo Zea indica el significado de esas labores del Seminario y del Instituto en el artículo La filosofía contemporánea en Latinoamérica ${ }^{2}$ cuando destaca:

\begin{abstract}
Este Seminario es importante porque ha reunido a filósofos y a científicos para que planteen $y$ discutan los diversos problemas que se presentan a unos y a otros mostrando la diversidad de enfoques que puede darse a los mismos y sus múltiples soluciones.

... y porque trabaja en América Latina sobre los grandes problemas de la filosofia tratando de darles una interpretación personal no por simple afán de originalidad, sino llevados por un auténtico espíritu filosófico, un afán de saber, el afán de saber que hace del Hombre, de cualquier hombre, los problemas centrales de la filosofía de cualquier latitud y tiempo.
\end{abstract}

Según Liliana Giorgis en Leopoldo Zea. Propuestas para la construcción de un futuro igualmente deseable para todos ${ }^{3}$, el Instituto Panamericano de Geografía e Historia se crea en 1927 por medio de la Organización de Estados Americanos y en cada país se establece una comisión que trabaja el desarrollo de las ideas. La presencia de Zea en la década de los años cincuenta en este seminario de las ideas, es fundamental para fijar la trayectoria del pensamiento y constituir la filosofía latinoamericana que nos lleva al día de hoy.

Indica Zea al respecto:

El primer problema planteado en este campo se refirió al de la posibilidad de una filosofía
americana que ha concluido en una afirmación positiva: la filosofía americana existe en
cuanto ha surgido el hombre preocupado por este problema y los problemas generales que
toda filosofía plantea. Apoyados en el historicismo, numerosos estudiosos de la filosofía en
la América Latina se han lanzado a la historia, al pasado, para buscar en ella las huellas que
la filosofía occidental ha dejado en esta América. Huellas en las que el mismo tiempo se ha
hecho patente la original interpretación de que fue objeto toda la filosofía al ser asimilada
por los pensadores de esta América con miras prácticas, para servir a la construcción del
ideal de la nacionalidad, de su ideal de convivencia política y social. ${ }^{4}$

En este marco de acontecimientos se celebra El Primer Seminario sobre Historia de las Ideas en América del 3 al 8 de diciembre de 1956 en San Juan de Puerto Rico; de tal forma la Isla se une al

\footnotetext{
${ }^{2}$ Leopoldo Zea," La filosofía contemporánea en Latinoamérica“ en : Filosofía y cultura latinoamericanas, Caracas, Venezuela, Centro Rómulo Gallegos 1976: 62-63.

${ }^{3}$ Liliana Giorgis, Estudios de Filosofía Práctica e Historia de las Ideas www.cricyt.edu.ar/estudios ,Revista anual de la Unidad de Historiografía e Historia de las Ideas - INCIHUSA / Mendoza, Año 7 / Nº 8 / ISSN 1515-7180 / Diciembre 2006 / Dossier (45-52)

${ }^{4}$ Zea, La filosofía contemporánea:64.
} 
proyecto del Instituto Panamericano de Geografía e Historia, incorporándose a esta temática investigativa estudiosos puertorriqueños como: Monelisa Lina Pérez Marchand y Domingo Marrero Navarro.

Los textos recopilados al momento sobre dicho seminario, son una serie de ponencias en la que participan personalidades de las Américas y el Caribe ${ }^{5}$. Coinciden los títulos de las ponencias con las recogidas en instituciones que tenían los textos como son la Fundación Francisco Manrique Cabrera y el Seminario Evangélico de Puerto Rico. El contenido de la revista difiere del conseguido por que algunas declaraciones como las palabras de presentación de Leopoldo Zea, la exposición sobre este primer seminario, la información sobre los ponentes y una carta del Dr. Juan Marín, no los hemos recibido. El resto del contenido está completo con los materiales encontrados:

Palabras de Apertura Arturo Morales Carrión *6

Arturo Ardao, Sobre el concepto de historia de las ideas *

Joao Cruz Costa, Historia de las ideas en el Brasil, subdesarrollo, influencias de Europa y Norteamérica y sus relaciones con la Historia de las ideas en la América Española**7

Benjamín Carrión, Historia de las Ideas en Ecuador*

Adolfo de Hostos, Apuntes para la Historia de las ideas en América: El culto de la conciencia de Hostos *

Diego Domínguez Caballero, Panorama de la historia de las ideas en Latinoamérica ** Antonio Gerbi, La visión europea en los pensadores americanos *

Domingo Marrero Navarro, Notas para organizar el estudio de las ideas en Puerto Rico**

Francisco Miró Quesada El filósofo europeo visto por el latinoamericano *

Pan-American Committee on the history of Ideas in American United States Sub Committee $* *$

Monelisa Pérez Marchand, Preámbulo para la historia de las ideas en Puerto Rico ** Humberto Piñera Llera, La historia de las ideas y la realidad americana *

Max Sarielle, La historia de las ideas en los Estados Unidos*

Santiago Vidal Muñoz, Panorama esquemático de la historia de las ideas en Chile.* Historia de las ideas como problema de la filosofía de la historia.*

Leopoldo Zea, América en la Historia*

\footnotetext{
${ }^{5}$ Según he investigado existe una publicación de este evento realizado por la Casa de la Cultura en Ecuador; solicité y recibí copia del índice por intercambio bibliotecario para posteriormente obtener la copia fotostática del número.

$6 *$ fotocopiado de la fundación Francisco Manrique Cabrera

$7 * *$ fotocopiado del Seminario Evangélico de Puerto Rico
} 
Estos títulos y conferenciantes de 1956, muestran la reflexión de los intelectuales de los dos continentes y el Caribe respecto al proceso de pensamiento en este otro lado del mundo junto al análisis realizado a las corrientes heredadas. Una de las aportaciones de dicho encuentro es la exposición de la síntesis de lo que será el futuro desarrollo de las teorías latinoamericanas, de los diálogos entre las culturas, sobre el proceso de pensamiento, las ideas y aportaciones filosóficas de los representantes de los continentes e islas; lo cual implica la reflexión sobre lo vuelto a hacer o hecho desde la realidad americana. Esta es la primera referencia que tenemos de una conferencia de Leopoldo Zea en Puerto Rico.

Coetáneo al seminario aparece un artículo suyo, "Hispanoamérica y el mundo occidental" en la Revista La Torre, de la Editorial de la Universidad de Puerto Rico en 1956. Años después participa con el ensayo "Hostos como conciencia latinoamericana" en la publicación que se hiciera para el Sesquicentenario de Eugenio María de Hostos en 1989 celebrada en la Universidad de Puerto Rico en Río Piedras, recogida en la publicación Hostos: sentido y proyección de su obra en América ${ }^{8}$. Otra referencia sobre su actividad con los estudios temáticos en Puerto Rico la obtenemos del programa para el Segundo Congreso Internacional de Filosofía Latinoamericana celebrado del 19 al 22 de octubre de 1993. Según el coordinador del evento Carlos Rojas Osorio, Leopoldo Zea no pudo asistir, aunque el título de la conferencia era Filosofar desde la marginación.

El título asignado a la actividad de 1993 fue Discurso desde la marginación y la barbarie 9 , años después Zea presenta su libro Emergencia de los marginados (2000); anteriormente su libro América en la historia se publicó en 1957; en ambas exposiciones presenta los trabajos que estaba realizando como una manera de recapitular y provocar al diálogo conducente a la reflexión del acontecer americano.

El ensayo presentado en 1956, América en la historia tiene dos partes: 'América como utopía' y la segunda parte, 'Incorporación de América en la Historia'. Al leer el referente encontramos que el texto coincide casi en su totalidad con las definiciones sobre "conciencia histórica", "utopía", “marginación histórica” del primer capítulo del libro homólogo, correspondiendo a las partes 4 y 5 de este capítulo inicial. El libro América en la Historia tiene como fecha de publicación el $1957^{10}$. Este dato nos ubica frente a varias consideraciones entre las cuales destaco la presentación de 1956 en Puerto Rico, porque expone una muestra del carácter y la reflexión sobre el ser latinoamericano, su proceso histórico y el pensamiento, en este momento de desarrollo de su concepto sobre América

\footnotetext{
${ }^{8}$ Leopoldo Zea, “ Hostos como conciencia latinoamericana“, en: Hostos : sentido y proyección de su obra en América, Instituto de Estudios Hostosianos, Editorial Universidad de Puerto Rico,1995:509-518.

${ }^{9}$ Leopoldo Zea, Discurso desde la marginación y la barbarie, Barcelona, Anthrophos: 1988.

${ }^{10}$ Leopoldo Zea, América en la Historia, Madrid, Editorial Revista de Occidente, 1957.
} 
Latina. Dicha exposición compara con lo heredado e impuesto en cuanto a conocimientos históricos y culturales que van delineando la conciencia americana y la identidad, en la discusión sobre la originalidad del lo hispanoamericano.

La conferencia muestra la temática que desarrollará en textos significativos para nuestro trabajo: La filosofía americana como filosofía sin más (1969); Filosofía y cultura latinoamericanas (1976); Filosofía de la historia Americana (1978); El problema de la identidad latinoamericana (1985).

Las publicaciones de Zea correspondientes a su responsabilidad y cargos, reflejan el trabajo realizado en diversos países latinoamericanos. Junto a estudiosos que forman parte del proyecto "Historia de las Ideas en América," las investigaciones se publican con la cooperación de la Comisión de Historia del Instituto Panamericano de Geografía e Historia y el Fondo de Cultura Económica de México. El prólogo de Filosofía de la historia americana de 1978 tiene la trayectoria del grupo de trabajo.

La lectura de las conferencias de 1956 muestran la pauta de relaciones entre los estudiosos: el diálogo entre distintos puntos de vista que mantendrá la discusión e investigaciones de esta generación de filósofos latinoamericanos aunque algunos de ellos se denominen sólo pensadores. Los títulos publicados mantienen las diversas opiniones, explicaciones y comparaciones sobre temas filosóficos respecto a su congruencia en América.

Zea señala en "La filosofía como originalidad"11 que: los sistemas filosóficos europeos han surgido ante una realidad cuyos problemas le han obligado a reflexionar, a pensar, a filosofar. Y para explicar este punto cita a Juan Bautista Alberdi: porque cada país, cada época y cada escuela ha dado soluciones distintas a los problemas del espíritu humano. Añade más adelante: La filosofía funciona como ideología, da razones, no del ser o de los entes, como algunas expresiones de la filosófica occidental, sino del orden político y social latinoamericano. ${ }^{12}$

Uno de los estudios sobre el filósofo mexicano, muestra la base de su pensamiento; al respecto cita Francisco Miró Quesada ${ }^{13}$ la explicación que hace sobre la conciencia del opresor- oprimido y el reconocimiento realizado por Zea, al exponer que en la diferencia yace la igualdad. Inicia ese proceso al centrar sus reflexiones en el ser humano como problema y proponer la igualdad en la diferencia. Ningún hombre es igual al otro y éste ser distinto es precisamente lo que le hace igual al otro, ya que como él posee su propia e indiscutible identidad. Ante la discusión de la posmodernidad que destruye

\footnotetext{
${ }^{11}$ Leopoldo Zea, "La filosofía como originalidad" en: La Filosofía americana como filosofía sin más México, Siglo XXI, 1969: 28-29.

12 Zea: 31.

${ }^{13}$ Francisco Miró Quesada, Leopoldo Zea, Madrid, Ediciones Orto, 1997.
} 
sin propuestas las nuevas creaciones, Gómez Martínez indica que Zea quiere superar este estado de confrontación. Como todos los hombres son iguales por ser diferentes, ningún pueblo puede considerarse civilizado mientras su estructura sociopolítica lleve consigo la opresión de otros pueblos.

Se reafirma Zea en este contexto y lo deja claro al interpretar magistralmente el ensayo “Nuestra América” de José Martí en las últimas páginas de Filosofía de la historia americana ${ }^{14}$. Al respecto dice: El desconocimiento de la propia historia, tanto el desconocimiento de las propias fuerzas y la admiración irracional de historias y fuerzas extrañas han podido conducir a algunos latinoamericanos a desear la subordinación para salir de otra. De allí la necesidad de conocer y asumir la propia historia; de conocer y asumir la propia realidad. Esta es la premisa que sostiene su convencimiento: el valor de conocer y estar consciente del quehacer de la libertad, que es la vía de la conciencia histórica.

El 27 de noviembre de 1967 en Lima, Perú continúa el programa de la Conferencia General de la UNESCO de 1966 para atender estudios culturales ${ }^{15}$. Se inscribe el programa América latina en su cultura. Los estudios de la región desde la arquitectura, las artes plásticas, la música, la literatura, la historia y las ideas constituyen las temáticas para proyectar el desarrollo de América Latina. Esta comisión eligió como presidente al gran filosofo mexicano Leopoldo Zea, que al respecto dijo: Sean del signo que fueren, los pensadores latinoamericanos están de acuerdo en afirmar la comunidad de destino de sus pueblos probada a lo largo de la historia que, al ser escrita, muestramás allá de enfoques parciales o sectoriales- su vocación unitaria. ${ }^{16}$ Bajo su dirección se trabaja en este proyecto que luego tiene la exposición en un programa de publicaciones de la UNESCO ${ }^{17}$ con el propósito de exponer la cultura latinoamericana en todos sus aspectos. Dice Zea en la Introducción que: Para librarse del mundo y cultura impuesta por el "descubrimiento" y la conquista españolas, la generación que sigue a la de los libertadores, se empeñará en lo que llamará "emancipación mental de esta América: ${ }^{18}$

Una de las aportaciones de Zea es cuando concurre en que: la filosofía de la liberación propugnada es en gran medida filosofía, que partiendo de la circunstancia que es la dependencia, trata de influir sobre esa realidad para transformarla hacia cada vez mayor independencia. ${ }^{19}$ Esa es la ruta trazada, que su vida intelectual elaboró para el beneficio de futuras generaciones y para contribuir en el entendimiento de los procesos, eventos y pensamientos marcados en la historia de los

\footnotetext{
${ }^{14}$ Leopoldo Zea, Filosofía de la historia americana, México, Fondo de Cultura Económica, 1978:289-294.

${ }^{15}$ Leopoldo Zea editor“ Prefacio“” en: América Latina en sus ideas, Siglo XXI, 1986:10.

16 "Prefacio", 1986:11.

${ }^{17}$ Publicaciones de la UNESCO: América Latina en su literatura; en sus artes; en su música, en sus ideas; en su historia, en su arquitectura. Dicha serie la publicó la Editorial Siglo XXI.

${ }^{18}$ Zea en:"Introducción“"17-18.

19*Prefacio" $: 13$.
} 
países sometidos, con el fin de recobrar su propio acontecer, superando las pautas establecidas por otros que han impuesto sus formas de vivir.

En el Homenaje a Leopoldo Zea: La Utopía de América, Simposio Internacional sobre el Quinto Centenario, en la Universidad Autónoma de Santo Domingo 1992, ${ }^{20}$ acto celebrado el 2 de octubre de 1992, el Mtro. Mario Magallòn Anaya de México, en su ponencia “Leopoldo Zea y la filosófica latinoamericana“ dijo:

Zea no plantea un modelo a seguir para alcanzar la libertad porque no hay modelos, más aun los modelos crean paternalismos y dictaduras en nombre de la libertad. Su postura es luchar por la libertad en la solidaridad y solidaridad en libertad, libertad que implica la defensa de los intereses populares ${ }^{21}$.

Concluye diciendo:.. la filosofía de Zea presta una doble función: por un lado en el de la sistematización y rigurosidad, y por el otro, en el de la función liberadora como actividad filosófica genuina. $^{22}$

Por esta reflexión, la búsqueda de la razón y el análisis es la constante para la toma de acciones. Esa indagación es la que establece en el capítulo "Búsqueda de la identidad latinoamericana“ en: El problema de la identidad latinoamericana ${ }^{23}$ estableciendo que no hay respuesta al momento, ni solución, de lo cual citamos: Entre una identidad impuesta y una identidad adoptada en América latina seguirá sin una definición de lo que podría ser su propia identidad. Encontramos que el trabajo Calibán $^{24}$ de Roberto Fernández Retamar contribuye a un detente para ponderar amplias reafirmaciones sobre la identidad latinoamericana.

La dialéctica de los temas latinoamericanos, en cuanto a la relación con los términos filosóficos que establecen las corrientes euro céntricas, tienen unas conclusiones que mantienen la trayectoria del quehacer filosófico desde las culturas náhuatl, maya, tojobal, quechua, mapuche, guaraní hasta la actualidad. Esta afirmación es una de la evidencias fundamentales del texto citado anteriormente, Pensamiento filosófico latinoamericano, del Caribe y “latino"(1300-2000) cuyos editores Enrique Dussel, Eduardo Mendieta y Carmen Bohórquez coordinan las investigaciones, análisis y correspondencias entre la cultura e historia de América Latina y las heredadas europeas para concluir con la hipótesis válida en toda la obra:

\footnotetext{
${ }^{20}$ Homenaje a Leopoldo Zea, La Utopía de América, Memorias Simposio Internacional sobre el Quinto Centenario, Universidad Autónoma de Santo Domingo 1992

${ }^{21}$ Mario Magallón Anaya, "Leopoldo Zea y la filosofía latinoamericana” en: La Utopía de América: 17.

22 Magallón Anaya: 20.

${ }^{23}$ Leopoldo Zea, El problema de la identidad latinoamericana, México, Universidad Nacional Autónoma de México, 1985: 23.

${ }^{24}$ Roberto Fernández Retamar, Calibán, los primeros ensayos son de 1977. La trayectoria del estudio la tenemos en la obra Todo Calibán, San Juan, Puerto Rico, Ediciones Callejón, 2003.
} 
... que la filosofía colonial no es meramente imitativa, sino que adoptando la Ilustración europea de la modernidad madura, la utiliza para sus propios fines, lo que exige por parte del investigador actual, y desde ahora, una nueva lectura de la historia de la filosófica latinoamericana, porque la importancia de esa filosofía no consiste en repetir o comentar temas de la filosofía europea del momento, sino para hacerlos funcionales a los intereses de los grupos dominantes de la periferia. ${ }^{25}$

Los diferentes pensamientos implican la dialéctica ${ }^{26}$ de la filosofía que continúa en el grupo de trabajo que representó Leopoldo Zea. A su vez parten de los análisis del Siglo XVII, XVIII y XIX que hicieran Sepúlveda, Bolívar, Rodríguez, Bello, Hostos y Martí entre otros.

Dicha trayectoria se traza en la aportación que hiciera Zea con motivo del sesquicentenario de Eugenio María de Hostos, la cual es significativa porque este puertorriqueño al igual que José Martí luchaba por la libertad de las Antillas, y tiene la base del pensamiento bolivariano, al establecer que nuevas repúblicas deben tener su cimiento en el derecho de la persona. Zea comenta y reflexiona en torno a los textos sobre Bolívar que Hostos escribe: 'Lo que intentó Bolívar', 'Ayacucho', 'Tres repúblicas'; en los comentarios encontramos el hilván que une el gran bordado cultural de América, con los diferentes colores, matices y texturas que implican las expresiones culturales de liberación.

\begin{abstract}
Bolívar no concebía la libertad de una nación de esta América sin la liberación del resto de las Naciones de la región. Dentro de la región estaban las naciones que el coloniaje español formó en las Antillas. Puerto Rico, la Dominicana y Cuba, son parte de toda una región cuya liberación depende de la totalidad de América Latina. Así lo recuerda Eugenio María de Hostos citando a Bolívar. El Libertador sabía que su obra no será completa sin la liberación de las Antillas, por ello trató de liberarlas una vez terminada la liberación del continente. Bolívar el 'hombre-legión', -dice Hostos- fue el primero que irrumpió el sueño de nuestra vida colonial para redimirnos. El hombre-idea fue el primero en concebir la patria inmensa y el que en cerebro ecuatorial nos hizo coeficiente de esa patria malograda. El hombrehumanidad fue el primero que, sin Cuba y sin Borinquén declaró incompleto el Continente y quiso abrasarnos en su fuego redentor: éramos para él pedazo de la humanidad que redimía.
\end{abstract}

Zea establece en este ensayo la trayectoria de sus estudios y definiciones del filosofar latinoamericano, al asentar su discurso en el reconocimiento de la igualdad humana en la diferencia. ${ }^{28}$ La interpretación de la obra de Hostos considerada en su contexto al igual que hace con Martí, lo llevan a una consideración sobre la situación política de Puerto Rico que es a su vez una situación de reflexión que atenta a la libertad de pensamiento, de reafirmación de identidad respecto de un presente para lograr un futuro.

Ayacucho fue la batalla de la liberación de América que se llamará latina: pero no fue la batalla decisiva. Lo que no hizo entonces Bolívar habrá que seguir haciéndolo. Los pueblos

\footnotetext{
${ }^{25}$ El pensamiento filosófico: 9.

${ }^{26}$ Leopoldo Zea, Dialéctica de la conciencia americana, México, Alianza Editorial Mexicana, 1976.

${ }^{27}$ Leopoldo Zea, Hostos como conciencia americana", en: Hostos: sentido y proyección de su obra en América: 514.

28 Gómez Martínez: 840.
} 

de esta América tienen que seguir luchando por su libertad y por su integración en la libertad; tienen que aún enfrentarse a quienes con diversos pretextos tratan de impedir el uso del derecho de esta América a la autodeterminación de sus pueblos. ${ }^{29}$

Por otro lado, Carlos Rojas en su libro Latinoamérica: cien años de filosofía expone que el término filosofía latinoamericana tiene dos sentidos: uno, como el quehacer discursivo de este continente $y$ el que designa una familia de tendencias que sostienen que hay una filosofía latinoamericana, y que se puede y debe hacer filosofía latinoamericana. ${ }^{30}$ Entendemos que este trabajo se debe en gran parte al esfuerzo de filósofos como Leopoldo Zea, quien insistió en hacernos ver nuestro pensamiento e identidad. A modo de resumen nos referimos al artículo sobre este filósofo mexicano que expone Rojas Osorio en su texto, el cual resume las interpretaciones de varios autores sobre la obra de Zea para concluir en que :

\begin{abstract}
Zea dirige la mirada hacia nosotros mismos, cambia radicalmente la perspectiva. La perspectiva se hace desde nosotros y hacia nosotros, no hay modelos abstractos. Todo pensamiento y toda valoración, y toda acción se proyectan y se realizan desde una determinada perspectiva. Sería una alineación permanente mantener esa mirada hipnótica hacia lo que es nuestro. Eso significa que nosotros tenemos que resolver nuestros propios planteamientos .......No es enajenarnos de la realidad mundial, sino entender la realidad mundial desde nuestra perspectiva. ${ }^{31}$
\end{abstract}

Las palabras de Leopoldo Zea nos relacionan con principios filosóficos como es la libertad, el derecho y la justicia, expuestos en el espejo de la realidad latinoamericana. En ambas intervenciones relacionadas con Puerto Rico dejó el camino definido para el quehacer del pensar, filosofar y hacer. Mediante su ejemplo metódico, atiende a las diferentes voces, en las que continúa la exégesis ante la necesidad del diálogo sobre, con y desde la idea de la libertad en nuestro espacio latinoamericano. Su trabajo semeja ser un faro que alumbra en las noches tranquilas y en las tormentosas; en ambas reconoce detalles que usualmente no observamos a la luz del día. Tal experiencia permite revaluar el pensamiento y las circunstancias para afrontar otros momentos y continuar con la vida en dignidad y equidad. La trayectoria de estudio y argumentación que hizo Leopoldo Zea como filósofo y maestro de generaciones ha permitido afianzar la conciencia histórica y el quehacer filosófico. Opino que su gestión ha valido para impulsar una mirada propia y pertinente, que se mantiene en los estudios actuales como son los problemas éticos y de la bioética contribuyendo, desde América Latina con interpretaciones pertinentes a los problemas de este lado del mundo; pero eso es tema para otro diálogo.

Muchas gracias.

\footnotetext{
29 Zea: 516.

${ }^{30}$ Carlos Rojas Osorio, Latinoamérica: cien años de filosofía, San Juan Puerto Rico , Isla Negra, 2002:11.

${ }^{31}$ Rojas Osorio.119-120.
} 


\section{BIBLIOGRAFIA}

Dussel,Enrique. Mendieta, Eduardo. Bohórquez,Carmen. Editores ,Pensamiento filosófico latinoamericano del Caribe y "latino“(1300-2000), México, Siglo XXI,2009.

Fernández Retamar, Roberto. Todo Calibán, San Juan, Puerto Rico, Editorial Callejón,2003.

Giorgis,Liliana. Estudios de Filosofía Práctica e Historia de las Ideas www.cricyt.edu.ar/estudios ,Revista anual de la Unidad de Historiografía e Historia de las Ideas - INCIHUSA / Mendoza, Año 7 / $\mathrm{N}^{\circ} 8$ / ISSN 1515-7180 / Diciembre 2006 / Dossier (45-52)

Gómez Martínez, José Luis. “Leopoldo Zea"en : Pensamiento filosófico latinoamericano del Caribe y“latino“(1300-2000), México, Siglo XXI,2009.

Homenaje a Leopoldo Zea, La Utopía de América, Memorias Simposio Internacional sobre el Quinto Centenario, Universidad Autónoma de Santo Domingo 1992.

Magallón Anaya, Mario. "Leopoldo Zea y la filosofía latinoamericana" en: La Utopía de América, Memorias Simposio Internacional sobre el Quinto Centenario, Universidad Autónoma de Santo Domingo 1992

Miró Quesada, Francisco. Leopoldo Zea, Madrid, Ediciones Orto, 1997.

Rojas Osorio, Carlos. Latinoamérica: cien años de filosofía, San Juan Puerto Rico, Isla Negra, 2002.

Zea, Leopoldo. América en la Historia, Madrid, Editorial Revista de Occidente,1957.

."La filosofía como originalidad" en: La Filosofía americana como filosofía sin más México, Siglo XXI, 1969.

.. La filosofía contemporánea en Latinoamérica“ en : Filosofía y cultura latinoamericanas, Caracas, Venezuela, Centro Rómulo Gallegos 1976.

. Dialéctica de la conciencia americana, México, Alianza Editorial Mexicana, 1976.

. Filosofía de la historia americana, México, Fondo de Cultura Económica, 1978.

. El problema de la identidad latinoamericana, México, Universidad Nacional Autónoma de México, 1985.

. Editor “ Prefacio“ en : América Latina en sus ideas, Siglo XXI, 1986.

Discurso desde la marginación y la barbarie, Barcelona, Anthrophos: 1988.

."Hostos como conciencia latinoamericana", en: Hostos : sentido y proyección de su

obra en América, Instituto de Estudios Hostosianos, Editorial Universidad de Puerto Rico,1995. 